\title{
Picturing Dengue in Vietnam
}

In August, 2019, Pearl Gan was invited to lead a dengue photography project at the Oxford University Clinical Research Unit (OUCRU), which works in partnership with the Hospital for Tropical Diseases, in Ho Chi Minh City, Vietnam. OUCRU is a centre of excellence for research into infectious diseases, and is one of the Wellcome Trust's five Africa and Asia Programmes. Dengue is a mosquito-borne viral disease that has a huge public health impact, with an estimated 390 million infections each year of which a quarter are symptomatic not only for Vietnam but also for the southeast Asian region and globally, and is a major research theme at OUCRU. There are currently no effective therapies for dengue and management relies on optimal supportive care. And as the only licensed vaccine, dengvaxia is limited for use in seropositive individuals, effective vector control strategies remains the most important approach in global disease prevention efforts.

These photographs highlight the impact of dengue on patients and aspects of the work of the dengue research group at OUCRU. Dr Sophie Yacoub leads the group, and a translational programme of clinical research focusing on pathogenesis studies, clinical trials of new therapeutics and innovative technology centred on non-invasive monitoring systems, , with the aim of improving the management and clinical outcomes of dengue patients. The group also works on mosquito-viral transmission dynamics to facilitate disease prevention strategies. This work builds on 20 years' of dengue research at OUCRU and HTD, previously led by Professors Bridget Wills and Cameron Simmons.

These images aim to raise awareness of the burden of dengue, as well as highlight the efforts made by the medical staff in local government hospitals to provide excellent clinical care in challenging settings and OUCRU's research efforts to improve clinical management and facilitate disease prevention strategies for Vietnam and globally.

Pearl Gan and Dr Sophie Yacoub

Oxford University Clinical Research Unit, Vietnam

www.pearlgan.com

www.OUCRU.org

All the photographs are by Pearl Gan. The words that accompany the photographs are by Pearl Gan and Sophie Yacoub. These photographs were taken in collaboration with the OUCRU Public Engagement department. I am indebted to Dr Sophie Yacoub (Head of the Dengue Research Group, OUCRU), Thuy Nguyen Thi Van (Research coordinator, Dengue group), Dr Mary Chambers (Head of Public Engagement, OUCRU), Professor Guy Thwaites (Director, OUCRU), and Dr Nguyen Van Vinh Chau (Director, HTD) and the OUCRU dengue research group. My personal inspiration for working with tropical diseases comes from Professor Kevin Baird (EOCRU). This work was funded by Wellcome [106680/Z/14/A]. 